\title{
Circulator Integrated in Low Temperature Co-fired Ceramics Technology
}

\author{
Raymond van Dijk, Gijs van der Bent \\ Dept. Radar Technology \\ TNO \\ The Netherlands \\ raymond.vandijk@tno.nl
}

\author{
Mohamed Ashari \\ Dept. Research and development \\ Via Electronic \\ Hermsdorf, Germany \\ m.ashari@via-electronic.de
}

\author{
Mark McKay \\ $R \& D$ \\ MESL Microwave \\ Edinburgh, United Kingdom \\ mark.mckay@meslmicrowave.com
}

\begin{abstract}
We present a demonstration of an integrated circulator for TR modules using low temperature co-fired ceramic (LTCC) technology. Two different circulators have been realised to be used in TR modules in two different frequency bands, $\mathrm{C}$ - and $\mathrm{Ku}$-band. The circulator is a three-port junction microstrip type device. The ferrite disc is embedded in the LTCC substrate and the composition is exposed to an external magnetic field, to provide the circulator as an integrated LTCC substrate circulator. Technological development, design and characterization of the circulator are reported.
\end{abstract}

Keywords-ceramics, ferrite, circulators, LTCC, TR modules

\section{INTRODUCTION ${ }^{1}$}

Microwave three-port circulators are crucial components in transmit-receive (TR) modules. In satellite systems TR modules are used in radar for earth observation and telecommunication equipment. The function of the circulator is the routing of the transmit and receive signals to the radiators and isolation of receivers and transmitter from the active reflection of array antennas. Depending on the requirements, a single or double circulator is used in each module.

Circulators are traditionally available as connectorised components or drop-in versions. Considering the requirements on size in TR modules for electronically steered array antennas connectorised modules are often prohibitive. For S-band and higher frequencies typical microwave connectors consume too much space. For these purposes drop-in circulators are preferred.

The connector from the drop-in circulator ferrite substrate to the TR module's circuit board is made by wire or ribbon bonds. These transitions are a crucial point in the multipaction susceptibility of the module because the circulator is situated in the high-power output path. The inevitable gap between the board edge and the drop-in circulator can form a cavity

\footnotetext{
${ }^{1}$ This work has been supported by the European Space
} Agency Basic Technology Research Programme. between the ribbon and ground plane. Multipaction can occur in this cavity, depending on dimensions, frequency and power levels.

By integration of the circulator in the module's circuit board multipaction problems can be minimised. Moreover the circuit board's mechanical stability is higher as the large window for mounting the drop-in can be avoided. Ceramic circuit boards, like low-temperature co-fired ceramics (LTCC) are a highperformance and cost effective technology with ample flight heritage.

In this paper we report on the integration of ferrites in the LTCC stack to build a circulator. The development of the technology and adaptations to the classical LTCC process flow, as well as circulator design and characterisation are reported. Two circulators, at $\mathrm{C}$-band and $\mathrm{Ku}$-band, have been made. Detailed multipaction analysis and test will be reported separately in a later publication.

\section{LTCC AND INTEGRATION TECHNOLOGY}

LTCC circuit boards are made starting from unfired ('green') ceramic sheets. These are punch and filled with metal paste, screen printed with metal traces and subsequently laminated together. This stack of layers with all traces is fired a low temperatures and then separate board are singulated by sawing or laser cutting.

The standard LTCC technology flow needs to be adapted to incorporate a suitable ferrite material. As the ferrite is relatively lossy for RF transmission purposes it should only be applied locally for the circulator function. In the vertical direction the ferrite cannot extend from the top to the bottom of the LTCC stack because the metallisation patterns for the circulator's resonator cannot cross from the LTCC top layer to the ferrite. This would cause disconnection during firing and would result in very cumbersome handling of green sheets of mixed materials. 
One approach to integrate circulators in LTCC is to use ferrite material in the 'green' state and sinter this together with the LTCC dielectric material [1]. This requires, from the mechanical point of view a ferrite material with low sintering temperature that is compatible with the LTCC sintering profile. It should also have identical shrinkage properties to reduce stress in the finished products. Microwave circulators require ferrite material with the right properties to make a good circulator. These requirements combined makes it very hard to find a proper material set. If it would be found or developed it would be a solution for a relatively narrow frequency regime as other frequencies require other ferrite properties (Section III).

We therefore pursue the path to integrate pre-sintered ferrite into LTCC cavities. The requirement on the ferrite, other than the electro-magnetic properties are that the height is an integer multiple of the fired LTCC layer thicknesses available for that material system.

The circulators have been realised using the DuPont 951 material system. They have been built from layers with a finished thickness of $216 \mu \mathrm{m}$ in a stack of either 9 or 5 layers, for $\mathrm{C}$-band and $\mathrm{Ku}$-band respectively.

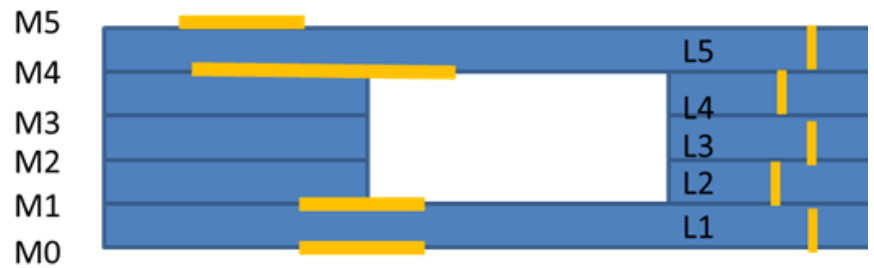

Fig. 1. Schematic of the layer stack with LTCC layers, metal traces, vias and cavity for the ferrite indicated.

Two layers of DuPont 951 PX are used to cover the ferrite on top and bottom in the C-band design. For Ku-band the total stack height would grow too large and thus a cover of one layer DuPont951 PX has been used on either side. The cover layers are needed for the application of the RF transmission lines around the ferrite and for mechanical stability. For the junction circulator it is important to have minimal non-ferrite material between the resonator and groundplane. Therefore metal traces are printed on the top of layer $L 1$ and on the bottom of layer L5. Because these layers also need metallisation on the outer surfaces for connection purposes there have been metallised on both sides (M0, MI and M4, M5).

The shrinkage of the LTCC layers has been determined accurately and the ferrite and cavity dimensions have been matched to have minimal stress in the finished device but without leaving a gap between the ferrite and LTCC edges. Shaping of the ferrite and LTCC cavity is such that a minimal amount of stress can be accommodated for.

The magnets for the static bias of the ferrite will be placed after LTCC sintering and singulation on the top and bottom of the outer layers.

\section{Circulator Design}

The demonstration of the technology has been realised starting from two sets of requirements, for the $\mathrm{C}$-band and $\mathrm{Ku}-$ band. The C-band device should have a return loss and an isolation of $<18 \mathrm{~dB}$ from 5.2 to $5.6 \mathrm{GHz}$. The $\mathrm{Ku}$-band device should have a return loss and an isolation of $<18 \mathrm{~dB}$ from 13.4 to $13.75 \mathrm{GHz}$.

A planar WYE resonator topology has been chosen to minimize the size of the ferrite disc and therewith minimize the potential stress after firing of the LTCC. Design equations are available for circulators that do not require very broadband operation [2]. These have been used to perform a first design and determine the required ferrite material, ferrite thickness and resonator dimensions (Fig. 2). Using the so obtained values the circulator geometry (see Fig. 3) has been optimised using a $3 \mathrm{D}$ finite element method electro-magnetic simulator, ANSYS HFSS.

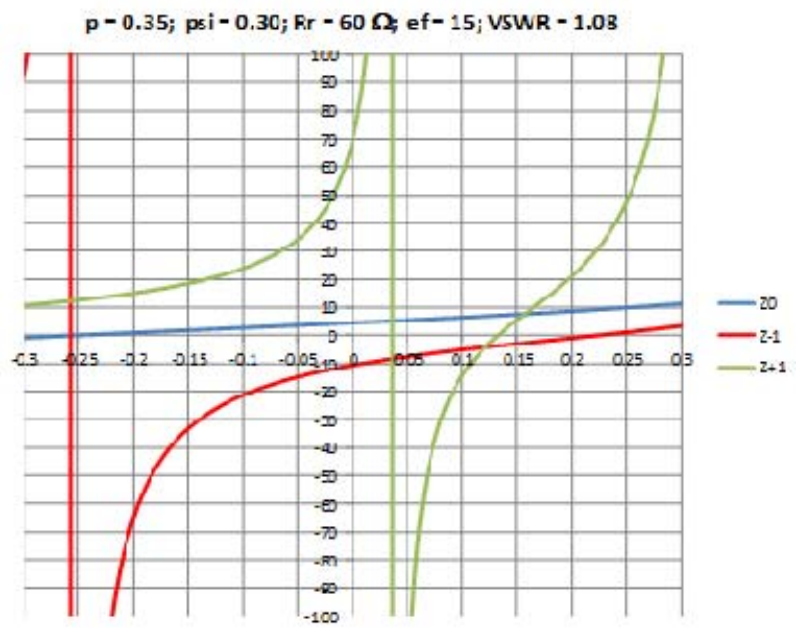

Fig. 2. Plot of the in-phase $\left(Z^{0}\right)$ and couter-rotating impedance eigenvalues $\left(Z^{+1}, Z^{-1}\right)$ for the listed design parameters [2].

For the C-band design a gadolinium doped garnet from Transtech, with a thickness of $1.1 \mathrm{~mm}$ has been used. For the $\mathrm{Ku}$-band design a magnesium ferrite from Transtech, with a thickness of $0.65 \mathrm{~mm}$ has been used.

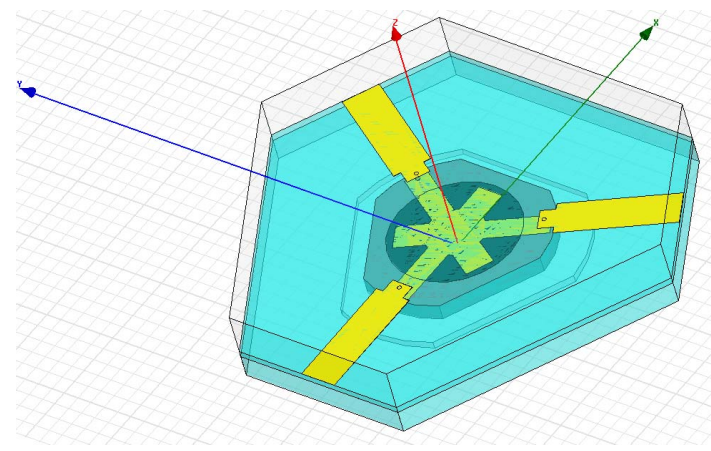

Fig. 3. Geometry used in the 3D software (Ansoft HFSS) for design optimisation.

Both devices are fitted with samarium-cobalt magnets of the appropriate size to define a uniform vertical magnetic field in the ferrite region of interest. Final tuning of the device has been performed by the exact location of the magnet. 


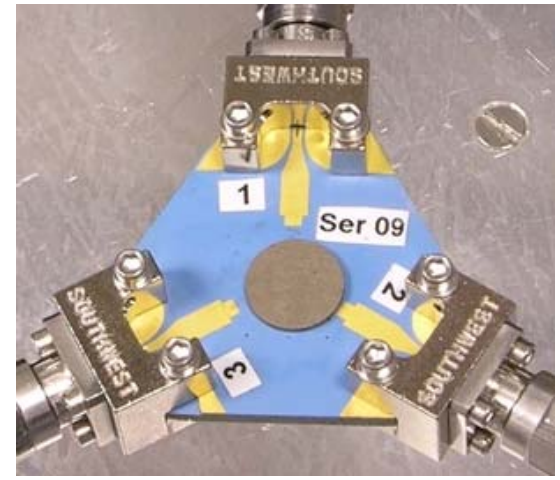

Fig. 4. C-band circulator, connectorised and magnets mounted.

A myriad of devices has been realized (Fig. 4), with different cavity dimensions (deliberate oversizing), variations on the serial matching line and parallel matching stub. The metal trace variations are meant to deal with process tolerances. The cavity variations are for multipaction testing.

\section{RF CHARACTERISATION}

Devices have been characterized at three temperatures. Small-signal measurements were performed directly while tuning the magnets and subsequently high-power measurements have been performed.

\section{A. Small Signal}

The small-signal properties of the devices is reported including the replaceable SMA test connectors and microwave launch with $50 \mathrm{Ohm}$ transmission lines up to the actual device. A loss of approx. 0.15 and $0.2 \mathrm{~dB}$ for C- and Ku-band respectively can be accounted for per interface.
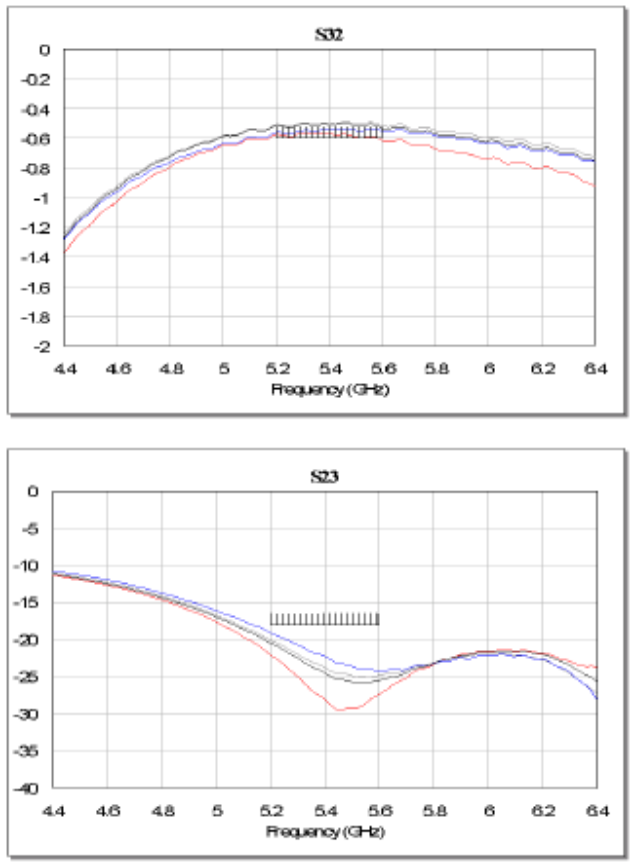

Fig. 5. Small-signal S-parameters for the C-band device. Red curve is $+80^{\circ} \mathrm{C}$, blue curve is $-20^{\circ} \mathrm{C}$ and grey and black are ambiant, before and after temperature cycle.
The loss of the C-band devices is therefore $0.3 \mathrm{~dB}$ or lower for all temperatures and frequency of interest (Fig. 5).
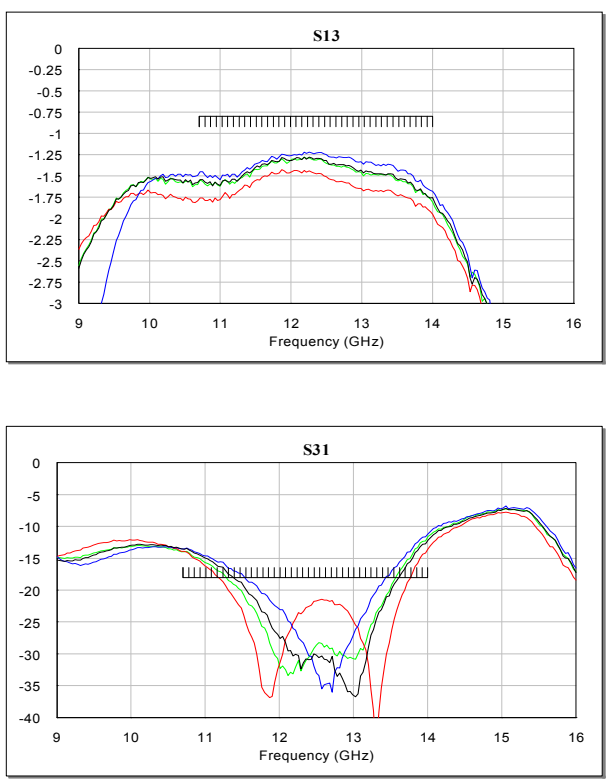

Fig. 6. Small-signal S-parameters for the Ku-band device. Red curve is $+80^{\circ} \mathrm{C}$, blue curve is $-20^{\circ} \mathrm{C}$ and green and black are ambiant, before and after temperature cycle.

The Ku-band devices have less than $1 \mathrm{~dB}$ loss after deembedding (Fig. 6). For both $\mathrm{C}$ - and Ku-band devices only the insertion loss and isolation are shown due to page restrictions. The input reflection is very similar to the isolation as can be expected for circulators.

\section{B. Power Measurements}

Power measurement has been performed $\mathrm{CW}$ and pulsed. For the CW case power has been applied to a port and insertion loss has been monitored at the extremes of the frequency band and for different port arrangements. A slight increase of loss with power is observed (see Fig. 7, Fig. 8). The effect is thermal as pulsed measurements with the same input power do not show any compression behavior, for duty cycles of 10 and $20 \%$. To test for the power compression of the device, the worst case situation appearing in a system need to be tested. This is the case when a maximum amount of signal is reflected back into the circulator. In this case constructive interference can cause the largest field strengths. The devices have been tested with the maximum power available from amplifiers at port 1 , and a full reflection with variable phase on the output port 2. The power out of port 3 is monitored. 


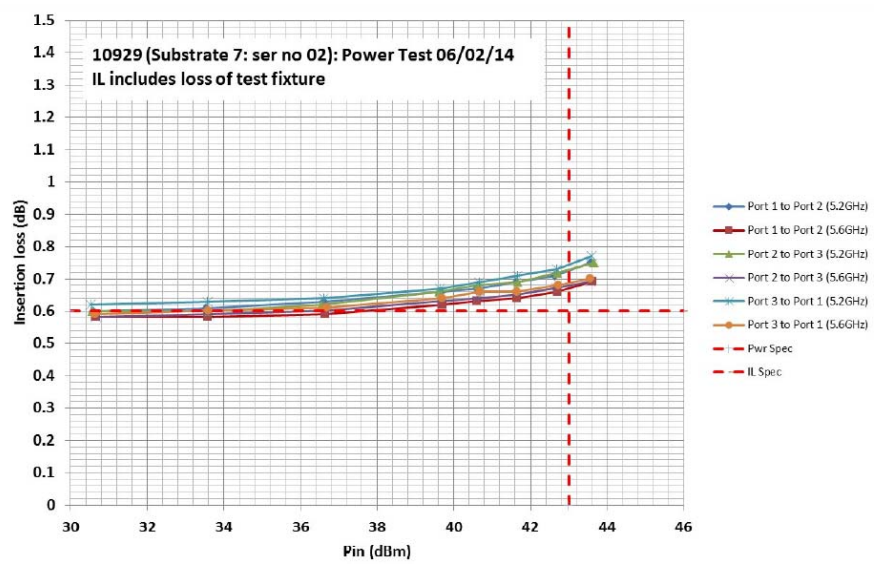

Fig. 7. Insertion loss under $\mathrm{CW}$ power conditions for a C-band device.

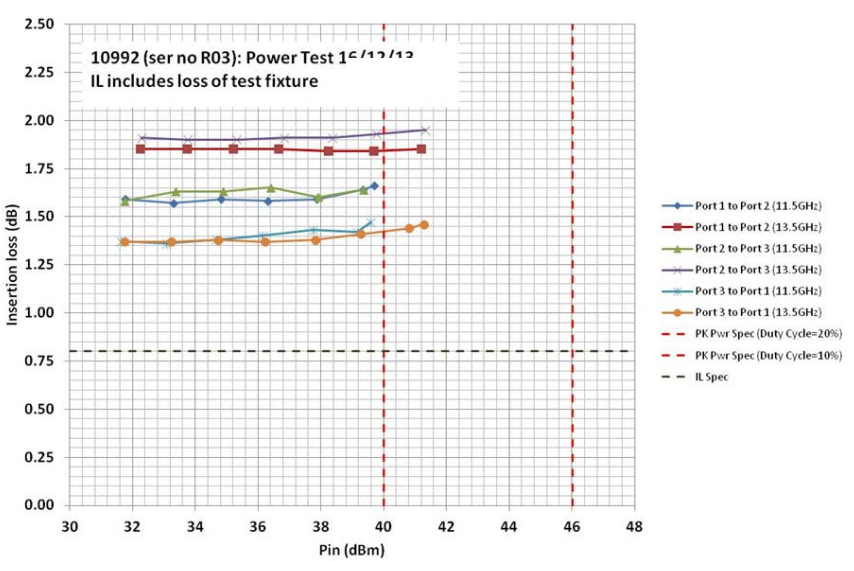

Fig. 8. Insertion loss under $\mathrm{CW}$ power conditions for a Ku-band device.

Taking into account the loss from the connectors and microstrip launches, the loss of the devices is within specification.

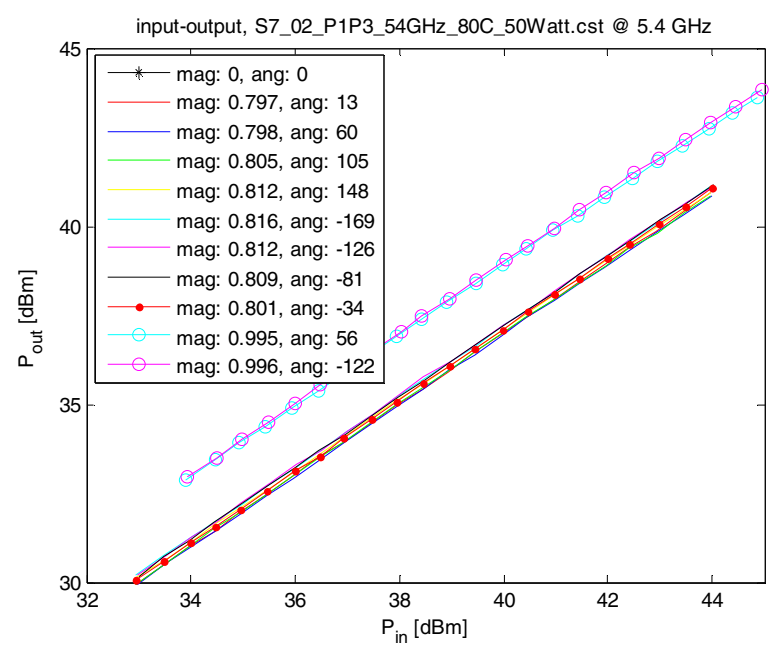

Fig. 9. Pin-Pout power handling curves for the C-band devices with a phase varying reflection at port 2 . Frequency is $5.4 \mathrm{GHz}$ and temperature is at $80^{\circ} \mathrm{C}$.

The power handling curves (Fig. 9) for the C-band device have been obtained with $50 \mu$ s pulses. The tuner on port 2 to reflect the signal with adjustable phase introduces some loss. To test with almost full reflection also a coaxial short and open standard are connected to the SMA connectors. This does give almost perfect reflection at the cost of having only two, noncontrolled, phase angles. The curves up to $45 \mathrm{dBm}$ show no sign of gain compression in the pulsed measurements. The behavior observed in the $\mathrm{CW}$ measurements is therefore attributed to thermal effects.

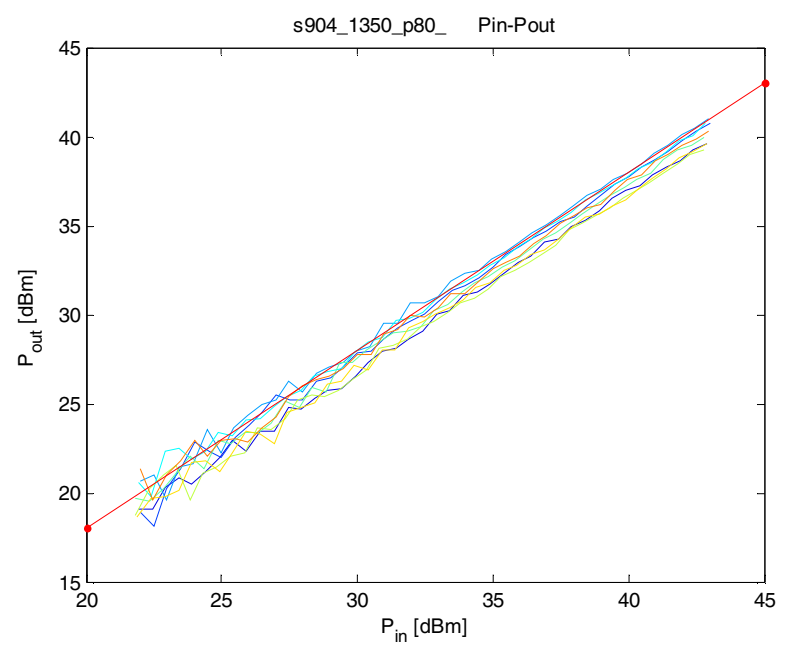

Fig. 10. Pin-Pout power handling curves for the Ku-band devices with a phase varying reflection at port 2 . Frequency is $13.5 \mathrm{GHz}$ and temperature is at $80^{\circ} \mathrm{C}$. Coloured lines are for varying reflection phase settings from $0-360^{\circ}$. A A fixed-loss line is drawn to assess (absence of) compression behaviour.

The input-output characteristics of the devices under full reflection show no compression up to $42.8 \mathrm{dBm}$ input power at the junction for $\mathrm{Ku}-\mathrm{band}$.

\section{CONCLUSIONS}

By using a hybrid technology approach we have demonstrated the integration of three-port junction circulators in an LTCC substrate. The technology is sufficiently controlled to have very tight fit of the ferrite in the LTCC host without introducing stress in the finished substrate. The properties of the circulators are as anticipated from the design which indicates that the LTCC technology is functionally unaffected by the inclusion of the pre-sintered ferrite. These demonstration devices show good power handling behavior up to $45 \mathrm{dBm}(\mathrm{C}$ band) and $43 \mathrm{dBm}$ (Ku-band). The integration of the circulator function in the substrate of a TR module leads to smaller and lighter module with more controlled characteristics with regard to multipaction.

\section{REFERENCES}

[1] T. Jensen, V. Krozer, and C. Kjærgaard. "Realisation of microstrip junction circulator using LTCC technology". Electronics Letters. 2011, 47(2). 111-113. Available: http://dx.doi.org/10.1049/el.2010.2419

[2] J. Helszajn, W.T. Nisbet, "Circulators using planar WYE resonators", IEEE Trans. MTT, Vol 29, No 7, July 1981 